EPJ Web of Conferences 32, 02006 (2012)

DOI: $10.1051 /$ epjconf/20123202006

(C) Owned by the authors, published by EDP Sciences, 2012

\title{
Progress of ECRH by EBW in over-dense plasmas and controlling the confinement regime by ECCD with high power launching in LHD
}

\author{
H. Igami ${ }^{1}$, Y. Yoshimura ${ }^{1}$, H. Takahashi ${ }^{1}$, T. Shimozuma ${ }^{1}$, S. Kubo ${ }^{1}$, H. Idei $^{2}$, M. Nishiura $^{1}$, S.

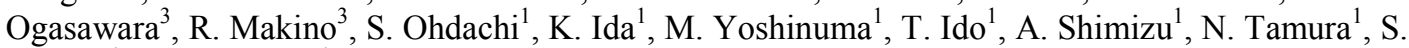 \\ Inagaki $^{1}$ and $\mathrm{T}$. Mutoh ${ }^{1}$ \\ ${ }^{1}$ National Institute for Fusion Science, 509-5292, Toki, Japan \\ ${ }^{2}$ Research Institute for Applied Mechanics, Kyushu Univ. 816-8580, Kasuga, Japan \\ ${ }^{3}$ Department of Energy and Technology, Nagoya Univ. Nagoya 464-8463, Japan
}

\begin{abstract}
In the large helical device (LHD), fundamental electron cyclotron resonance heating $(\mathrm{ECRH})$ by the electron Bernstein wave (EBW) excited via the ordinaryextraordinary-EBW (O-X-B) mode conversion process was performed with high power ( 1MW) launching. Profiles of increase of the electron temperature $\left(T_{e}\right)$ and the soft Xray signals during the power injection suggest power absorption in the core region. Effects of the local modification of the rotational transform $t / 2 \pi(=1 / q)$ by electron cyclotron current drive (ECCD) on the formation and sustainment of the electron internal transport barrier (e-ITB) was investigated for the first time. Co ECCD raised $\imath / 2 \pi$ close to 0.5 in the core region and caused the flattening of the $T_{e}$ profile. Additional ECRH power is required to form the e-ITB. On the contrary, counter (cntr.) ECCD separates $t / 2 \pi$ from 0.5 in the core region and avoids the flattening of the $T_{e}$ profile. The e-ITB can be formed and sustained without additional ECRH. Analysis of the heat pulse transport with use of the modulation ECRH (MECH) shows the good confinement region extends to the $l / 2 \pi=0.5$ rational surface in the case of cntr. ECCD.
\end{abstract}

\section{Introduction}

For ECRH in LHD, three $77 \mathrm{GHz}$ high power ( 1MW) gyrotrons have been installed continuously and contributed to improve the plasma performance. One of these $77 \mathrm{GHz}$ gyrotrons is installed in a transmission line that connects to the horizontal port antenna. The horizontal port antenna has a large flexibility of the toroidal launching angle therefore is available for excitation of the EBW via the O-X-B mode conversion process. The evidence of the EBW heating was obtained more clearly than the previous experiment [1]. With use of the same antenna, ECCD was also performed with high power. It was found that the local modification of the rotational transform $l / 2 \pi$ $(=1 / q)$ profile by ECCD affects the formation of the e-ITB for the first time.

In this paper, recent status of the ECRH system in LHD is introduced first in section 1.1. Then, recent progress of EBW heating via the O-X-B mode conversion process in LHD is reported in section 2 . In section 3 , effects of the local modification of the $/ 2 \pi$ profile by co and cntr. ECCD on the formation of the e-ITB are reported. In section 4, we summarize the contents. 


\subsection{Recent status of ECRH system in LHD}

Since 2006, we have developed and installed three $77 \mathrm{GHz}$ high power gyrotrons continuously under the collaboration with University of Tsukuba. In 2009, the newest gyrotron was installed. In 2010 , in the conditioning operation, the newest gyrotron achieved 1.76MW MOU output power for $0.7 \mathrm{~s}$ by two steps start-up of the anode voltage and also achieved $0.31 \mathrm{MW}$ MOU output power in a long pulse operation. In the last experimental campaign carried out in 2011 with five gyrotrons including one $82.7 \mathrm{GHz}$ and one $84 \mathrm{GHz}$ gyrotrons, the total input power into LHD achieved 3.78MW at maximum. In stable operations in short pulse discharges $(\sim 2 \mathrm{~s}$.) $3.3 \mathrm{MW}$ could be launched in total. For each transmission line of $77 \mathrm{GHz}, 1 \mathrm{MW} / 2 \mathrm{~s}$ power injection to LHD was stably available. In the long pulse experiment, ECR plasma of $\mathrm{T}_{\mathrm{e}}=2 \mathrm{keV}, \mathrm{n}_{\mathrm{e}}=1.5 \times 10^{19} \mathrm{~m}^{-3}$, was sustained for $60 \mathrm{sec}$. with $0.592 \mathrm{MW}$ power injection with three $77 \mathrm{GHz}$ and one $84 \mathrm{GHz}$ gyrotrons. The transmission lines for $77 \mathrm{GHz}$ gyrotrons which consist of $88.9 \mathrm{~mm}$ diameter waveguides are evacuated to avoid arcing.

\section{Recent progress of the EBW heating via the O-X-B mode conversion process in LHD}

\subsection{Methods of the EBW excitation in LHD}

With the development of the high density operation e.g. the formation of the super dens core (SDC) plasma in LHD, [2] study of ECRH by the EBW has grown in importance. Because the electron density at the electron cyclotron resonance (ECR) layer exceeds the cutoff density of the electron cyclotron (EC) wave in high density operations and in such over-dense plasmas the conventional ECRH by normal electromagnetic modes cannot be available. The EBW is a kind of electrostatic waves that propagates in magnetized plasmas. It has no density limit in propagation and is absorbed by electron cyclotron damping. Therefore ECRH by EBW has been expected as a possible substitute for conventional ECRH. However, the EBW is required to be excited via the mode conversion process from the slow X (SX)-mode in the upper hybrid resonance (UHR) layer therefore the electromagnetic wave should be injected from the outside of the plasma so that it connects to the SX-mode inside the plasma. In LHD, three kinds of excitation/heating method of the EBW have been studied. The direct oblique launching of the fundamental SX-mode from the high field side without additional mirror [3], the SX-mode launching from the high field side with use of an additional mirror installed on the vacuum vessel wall near the plasma surface [4], and the O-mode launching from the low field side $[1,5]$.

The O-mode launching has a great advantage that the launcher of the EC waves can be placed in the low field side where the space can be reserved easily apart from the plasma surface and has been studied continuously in LHD from the perspective of application in future fusion devices. On the other hand, the launching angle is restricted within the "mode conversion window" because the O-mode should be injected so that the parallel refractive index $N_{/ /}$is close to the optimum value $N_{/ / \text {opt }}$ at the plasma cutoff. If $N_{/ / /}=N_{/ / \text {opt }}=\{\beta /(1+\beta)\}^{1 / 2}$ at the plasma cutoff, where $\beta=\Omega_{\mathrm{ce}} / \omega, \Omega_{\mathrm{ce}}$ is the electron cyclotron angular frequency and $\omega$ is the wave angular frequency, the position of the plasma cutoff and the left handed cutoff coincide with each other and the incident O-mode connects to the $\mathrm{SX}$-mode. In the case, the SX-mode propagates toward the UHR layer and is mode converted to the EBW [6]. If $N_{/ /}$at the plasma cutoff deviates from $N_{/ / o p t}$, the evanescent region appears between the plasma cutoff and the left handed cutoff. However, if the evanescent region is thin, a part of the incident O-mode can transmit through the region and connects to the SX-mode then be mode converted to the EBW. The transmission rate $\mathrm{T}_{\mathrm{OX}}$, is given as the following equation [7].

$$
\mathrm{T}_{\mathrm{OX}}=\exp \left\{-\pi k_{0} L_{n}(\beta / 2)^{1 / 2}\left[2(1+\beta)\left(N_{/ /} N_{/ / / p t}\right)^{2}+N_{v}^{2}\right]\right\}=\mathrm{T}_{\text {OXB }}
$$


Where, $k_{0}$ is vacuum wave number, $L_{n}$ is the scale length of the density gradient, $N_{v}$ is the refractive index perpendicular to both of the magnetic field and the density gradient. Since the SX-mode is fully mode converted to the EBW in the linear theory, this transmission rate means the mode conversion rate from the incident $\mathrm{O}$-mode to the EBW via the X-mode, $\mathrm{T}_{\mathrm{OXB}}$. In LHD, $k_{0} L_{n}$ of eq. (1) is larger than 30, therefore little deviation of $N_{/ /}$from $N_{/ / \text {opt }}$ is allowed to obtain enough mode conversion rate. Previous numerical estimation suggests that the width of the mode conversion window of the launching angle where $\mathrm{T}_{\mathrm{OXB}}>0.5 \mathrm{can}$ be obtained is $0.4 \mathrm{deg}$. In the experiment in LHD, the O-mode is launched toward this narrow mode conversion window in over dense plasmas.

\subsection{Experimental results of the O-mode launching to the "over-dense" plasmas}

For the experiment of the EBW heating via the O-X-B mode conversion process, a magnetic configuration $\left(\mathrm{R}_{\mathrm{ax}}, \mathrm{B}_{\mathrm{t}}\right)=(3.75 \mathrm{~m}, 2.4 \mathrm{~T})$ was selected, where $\mathrm{R}_{\mathrm{ax}}$ is the position of the magnetic axis and $\mathrm{B}_{\mathrm{t}}$ is the magnetic field strength at the magnetic axis. The $77 \mathrm{GHz} \mathrm{EC}$ wave was injected with the polarization of the left-handed circular polarized wave that is almost the O-mode, toward the vicinity of the "mode conversion window" predicted by the previous numerical investigation. The launching angle was changed shot by shot to search the good one with checking the effect of the ECRH. Fig.1 shows waveforms of a discharge. The target helium plasma was sustained by neutral beam injection (NBI) and the line averaged electron density at the plasma centre exceeds the plasma cutoff density. Increase of the stored energy and the electron temperature measured by electron cyclotron emission (ECE) radiometer were observed during the EC wave injection. The temporal differentiation of the soft X-ray signals increase after the ECRH turned on. This fact suggests the change of increase rate of the soft X-ray signal reflects the increase of the electron temperature. Note that the rapid increases of the soft X-ray signals at $4.55 \mathrm{~s}$ suggest the contamination by impurity. The absorbed power was estimated from the change of the temporal differentiation of the stored energy as $P_{a b s}=$ $\Delta\left(d W_{p} / d t\right)$ at the timings of turning on/off of the ECRH. The power absorption rate $P_{a b s} / P_{\text {in }}=0.12$ was obtained on an average. Where $P_{a b s}$ is the launched power.

In Fig.2, increase of the electron temperature, $\Delta T_{e}$ after the power injection for $0.1 \mathrm{~s}$ in consideration of the time trend is plotted versus the normalized minor radius $(\rho)$. The difference between the temporal differentiations, $\Delta\left(d T_{e} / d t\right)$ before and after turning on the ECRH are also plotted in the same graph. In Fig. 3, profiles of the increase of the soft X-ray intensity normalized by the soft X-ray intensity before turning on the ECRH, $\Delta S / S$ and the temporal differentiations normalized by the soft X-ray intensity before ECRH in consideration of the time trend, $\Delta(d S / d t) / S$ are plotted versus the normalized minor radius where the line of sight across the mid-plane ( $\rho_{\text {_mid- }}$ plane). The peak positions of $\Delta S / S$ and $\Delta(d S / d t) / S$ observed around $\rho_{\text {mid-plane }}=0.55$ are near the peak positions of $\Delta T_{e}$ and $\Delta\left(d T_{e} / d t\right)$ in Fig. 2 .
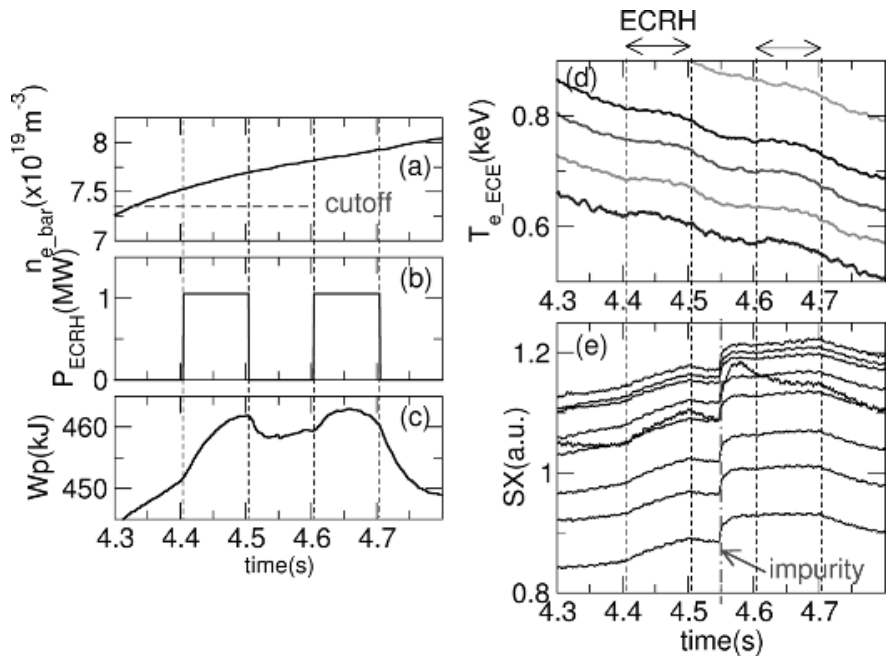

02006-p.3
Fig.1: (Discharge \#106297) (a) : Line averaged electron density, (b) : ECRH power (c) : Stored energy (d) : Electron temperature measured by ECE radiometer (e) : soft X-ray signals 


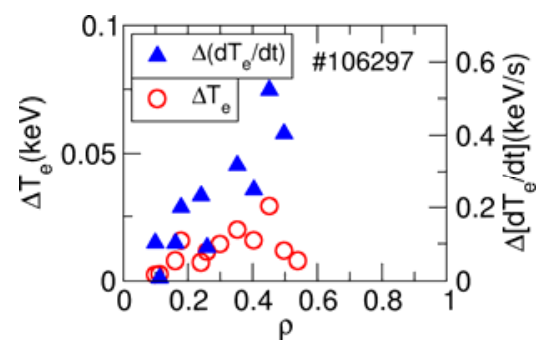

Fig.2 : Profiles of the increase of the electron temperature after $0.1 \mathrm{~s}$ power injection in consideration of the time trend and the difference between the temporal different-tiations before and after turning on the ECRH. The magnetic configuration $\left(\mathrm{R}_{\mathrm{ax}}, \mathrm{B}_{\mathrm{t}}\right)=(3.75 \mathrm{~m}, 2.4 \mathrm{~T})$ was selected.

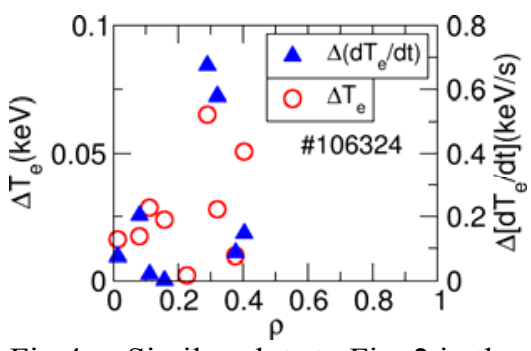

Fig.4 : Similar plots to Fig. 2 in the case of $\left(\mathrm{R}_{\mathrm{ax}}, \mathrm{B}_{\mathrm{t}}\right)=(3.75 \mathrm{~m}, 2.2 \mathrm{~T})$.

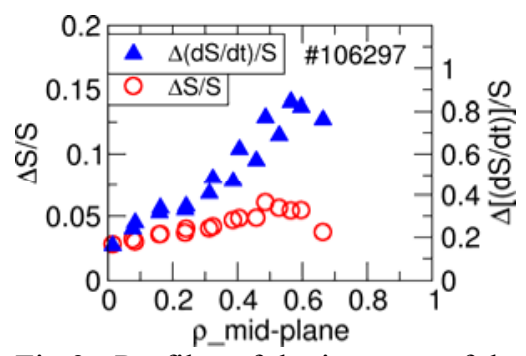

Fig.3 : Profiles of the increase of the normalized soft X-ray intensity $(\Delta \mathrm{S} / \mathrm{S})$ after $0.1 \mathrm{~s}$ power injection in consideration of the time trend and normalized difference between the temporal differentiations before and after turning on the ECRH. The magnetic configuration $\left(\mathrm{R}_{\mathrm{ax}}, \mathrm{B}_{\mathrm{t}}\right)$ $=(3.75 \mathrm{~m}, 2.4 \mathrm{~T})\left(\mathrm{R}_{\mathrm{ax}}, \mathrm{B}_{\mathrm{t}}\right)=(3.75 \mathrm{~m}, 2.4 \mathrm{~T})$

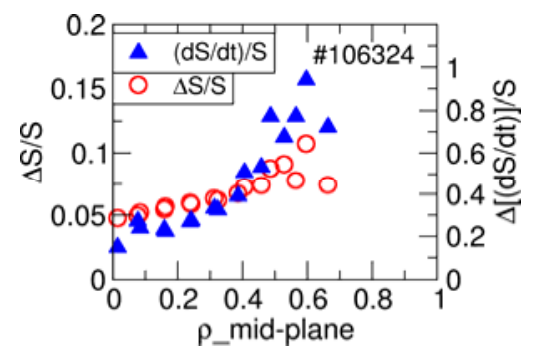

Fig. 5 : Similar plots to Fig. 3 in the case of $\left(\mathrm{R}_{\mathrm{ax}}, \mathrm{B}_{\mathrm{t}}\right)=(3.75 \mathrm{~m}, 2.2 \mathrm{~T})$.

For a weaker magnetic configuration of $\left(\mathrm{R}_{\mathrm{ax}}, \mathrm{B}_{\mathrm{t}}\right)=(3.75 \mathrm{~m}, 2.2 \mathrm{~T})$, increase of the stored energy was also observed during the EC wave injection in similar discharges. The power absorption rate was 0.09 on an average. In Fig. 4, profiles of $\Delta T_{e}$ and $\Delta\left(d T_{e} / d t\right)$ are plotted. In fig. 5 Profiles of $\Delta S / S$ and $\Delta(d S / d t) / S$ are plotted. Near the magnetic axis, increase of $T_{e}$ and $d T_{e} / d t$ were observed. There are not channels of ECE to observe the region $\rho>0.4$. However peaks of $\Delta S / S$ and $\Delta(d S / d t) / S$ are shown around $\rho_{\text {mid-plane }}=0.6$. There is a possibility of the power absorption where $\rho>0.4$.

\subsection{Discussions}

The electron density profile was obtained by the Thomson scattering measurement with use of the calibration factor given by the millimeter wave interferometer that has the same line of sight as the Thomson scattering measurement. The plasma cutoff of the $77 \mathrm{GHz}$ EC wave was located close to the plasma edge. In both cases of $\left(\mathrm{R}_{\mathrm{ax}}, \mathrm{B}_{\mathrm{t}}\right)=(3.75 \mathrm{~m}, 2.4 \mathrm{~T})$ and $\left(\mathrm{R}_{\mathrm{ax}}, \mathrm{B}_{\mathrm{t}}\right)=(3.75 \mathrm{~m}, 2.2 \mathrm{~T})$ power absorption occurred in the core region where the normal modes of electromagnetic wave cannot access. ECRH by EBW excited via the O-X-B mode conversion process is a possible mechanism. The region where $T_{e}$ increased shifted toward the magnetic axis as $\mathrm{B}_{\mathrm{t}}$ decreased. This tendency is consistent with the results of the previous numerical prediction [8]. However the absorption regions are more inner side than the prediction. Previous numerical prediction suggests that the wall of the horizontal port hides the mode conversion window of high mode conversion rate. In our experiment, the EC wave was launched toward the "mode conversion window" of lower mode conversion rate.

Numerical analysis with the ray-tracing with use of the real experimental density and temperature profiles are required to determine the power deposition region and heating efficiency. Procedure to reconstruct the electron density profile near the plasma edge should be improved to calculate the O$\mathrm{X}$-B mode conversion rate to compare the position of the mode conversion window given in the numerical calculation with that suggested in the experiment. 


\section{Effects of the local modification of the rotational transform by ECCD on the formation and sustainment of the e-ITB}

\subsection{Introduction}

In helical systems, e-ITB formation has been explained in association with the transition of the radial electric field from negative (ion root) to positive (electron root) and has been induced by centrally focused ECH in plasmas. In LHD e-ITB has been observed with centrally focused ECH in plasmas sustained by counter (cntr.) NBI or only ECH before [9,10]. In LHD, near the $l / 2 \pi(=1 / \mathrm{q})=0.5$ rational surface, the $m / n=2 / 1$ magnetic island is formed. When it grows, confinement in the core region degrades and formation of the e-ITB may be affected. Local modification of the $l / 2 \pi$ profile is available with high power ECCD. Effect of the local modification of the $l / 2 \pi$ profile on the formation and sustainment of e-ITB is investigated by co and cntr. ECCD.

\subsection{Experimental results of e-ITB formation with co/cntr. ECCD}

In the experiment, a magnetic configuration $\left(\mathrm{R}_{\mathrm{ax}}, \mathrm{B}_{\mathrm{t}}\right)=(3.6 \mathrm{~m}, 2.75 \mathrm{~T})$ was selected. From $3.3 \mathrm{~s}$ to $4.8 \mathrm{~s}$ the target plasma was sustained by balanced tangential neutral beam injection (NBI), co/cntr. ECCD and modulation ECRH (MECH). The launched power for ECCD was about 1MW and that for $\mathrm{MECH}$ was about $0.5 \mathrm{MW}$. The line averaged electron density was sustained around $0.7 \sim 0.75$ $\mathrm{x} 10^{19} \mathrm{~m}^{-3}$ to avoid the effect of non-thermal electrons on the ECE. Fig. 6 shows the time evolution of the $T_{e}$ profile obtained by Thomson scattering measurement and $t / 2 \pi$ profile obtained by motional Stark effect (MSE) measurement [11] when co ECCD was applied. In the early phase of the discharge $(\mathrm{t}=3.5 \mathrm{~s})$ e-ITB is observed. However, $T_{e}$ near the magnetic axis suddenly dropped at $4.05 \mathrm{~s}$ and flat $T_{e}$ profile was observed $(\mathrm{t}=4.1 \mathrm{~s})$. The power absorption profile of co ECCD estimated by ray-tracing calculation with use of the dispersion equation in the hot plasma indicates the current is driven near the magnetic axis. Actually $l / 2 \pi$ inside $l / 2 \pi=0.5$ rational surface increased and approached to 0.5. During the balanced NBI and co ECCD for two seconds, the e-ITB was not formed unless the additional ECRH of $1 \mathrm{MW}$ was applied from $4.3 \mathrm{~s}$. The gradient of the plasma potential profile measured by heavy ion beam probe (HIBP) [12] was moderate in the region of the flat $T_{e}$ profile and it became steep after the e-ITB was formed again. Because of the balanced NBI, the effect of NB driven current on the modification of $l / 2 \pi$ profile is negligible. Fig. 7 shows the time change of the $T_{e}$ and $t / 2 \pi$ profiles when cntr. ECCD was applied. The e-ITB was observed in the early phase of discharge $(\mathrm{t}=3.5 \mathrm{~s})$ and sustained through the discharge without additional ECRH.
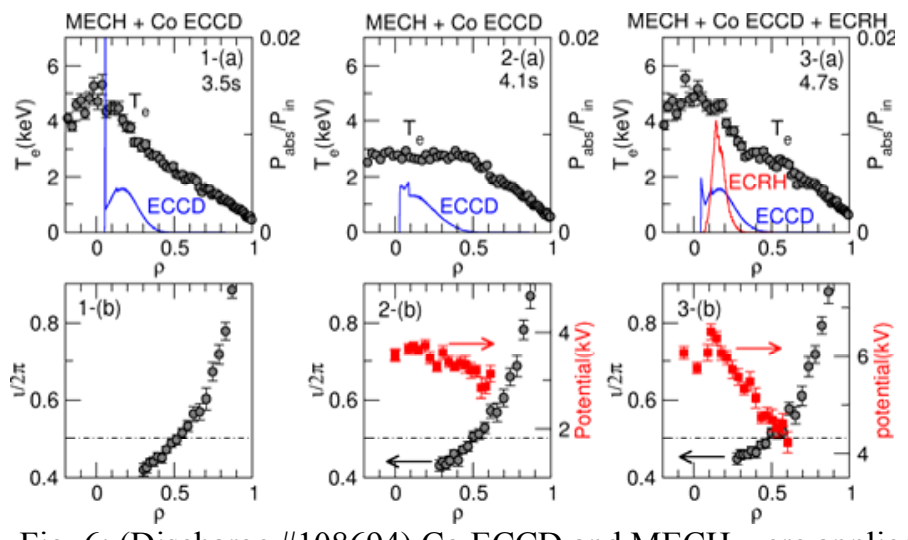

Fig. 6: (Discharge \#108694) Co ECCD and MECH were applied during the 2.5s discharge. 1,2,3-

(a): Profiles of the electron temperature profile $\left(T_{e}\right)$ and power absorption of ECCD and additional ECRH estimated by ray-tracing calculation. 1,2,3-(b): Profiles of the rotational transform $(l / 2 \pi)$ and the plasma potential. 

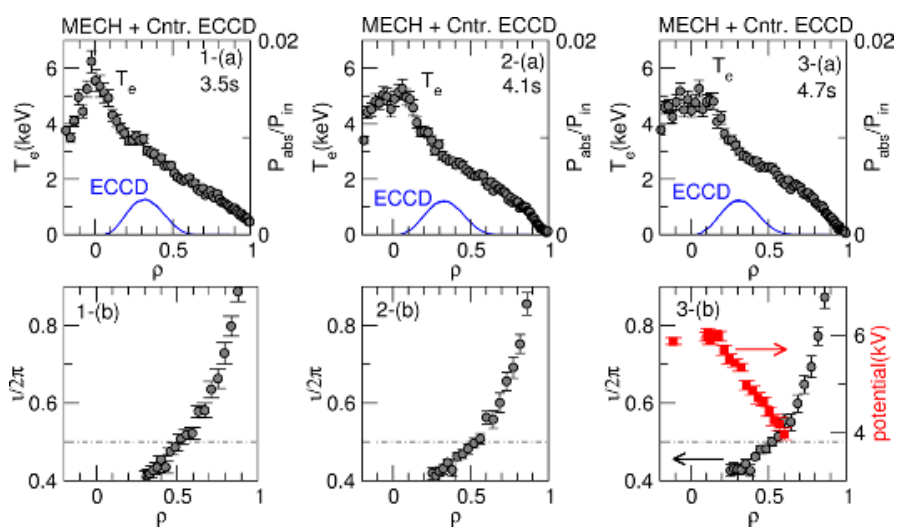

Fig. 7: (Discharge \#108690) Cntr ECCD and MECH were applied during the $2.5 \mathrm{~s}$ discharge. 1,2,3-(a): Time change of $T_{e}$ profile and power absorption profile of ECCD and estimated by raytracing calculation. (b): Time change of $l / 2 \pi$ and plasma potential profile.
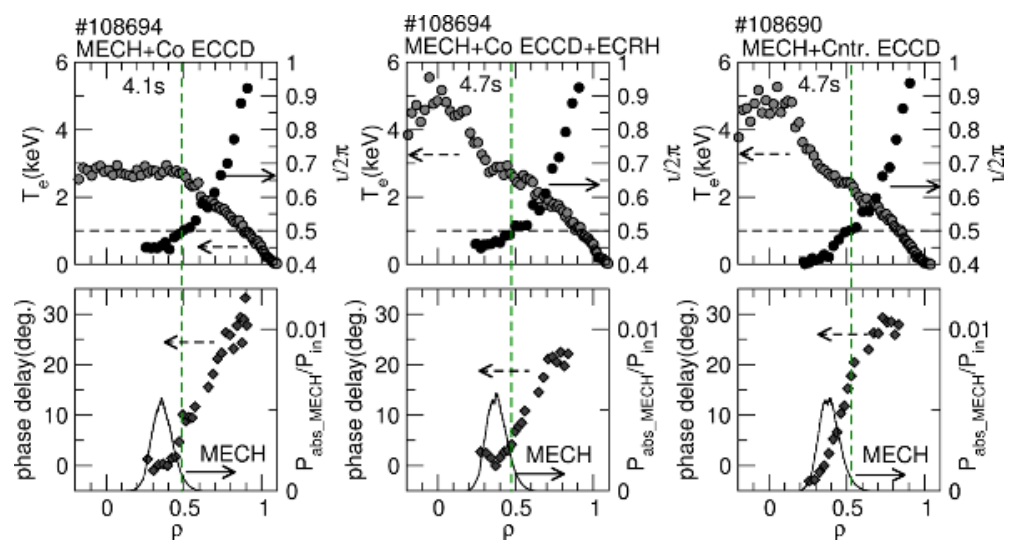

Fig. 8 : Profiles of the electron temperature and the rotational transform (upper). Profiles of the phase delay of $25 \mathrm{~Hz}$ heat pulse and the power absorption region of $\mathrm{MECH}$ (lower).

With time, $l / 2 \pi$ inside $l / 2 \pi=0.5$ rational surface is separated from 0.5 .

Characteristics of the heat pulse propagation were investigated by MECH. In each graph in the lower row in Fig.8, phase delay of the heat pulse of $25 \mathrm{~Hz}$ obtained by FFT analysis is plotted versus the normalized minor radius $\rho$ for each case of MECH+Co ECCD, MECH+Co ECCD + ECRH and $\mathrm{MECH}+\mathrm{Cntr}$. ECCD. Inside $\rho=0.25$ the phase delay is not plotted because fluctuations appear in ECE signals and the FFT analysis is not appropriate there. When the flat $T_{e}$ profile was observed during co ECCD inside the $l / 2 \pi=0.5$ rational surface (see \#108694, 4.1s) the change of the phase delay there was little. When the e-ITB was formed again by the additional ECRH (see \#108694, $4.7 \mathrm{~s}$ ), the local flattening of the $T_{e}$ profile was shown in the region of $0.25<\rho<0.5$ inside the $l / 2 \pi=$ 0.5 rational surface and the change of the phase delay there was little. On the contrary, in the case of $\mathrm{MECH}+$ cntr. ECCD (see \#108690, 4.7s), the local flattening of the $T_{e}$ profile was not observed in the region and the change of the phase delay is larger than the previous cases inside the $t / 2 \pi=0.5$ rational surface.

\subsection{Discussions}

By co ECCD, $l / 2 \pi$ was raised up close to 0.5 in the core region and the flat $T_{e}$ profile was observed. The flat $T_{e}$ profile and the $t / 2 \pi$ profile of moderate gradient (weak magnetic shear) close to 
driven by NBI [13]. In Ref. [13], it was pointed out that the global stochastization of the magnetic island occurred and caused the flattening of the $T_{e}$ profile. In our case, the global stochastization with flattening of the $T_{e}$ profile might be caused by non-inductive co ECCD. After the additional ECRH, e-ITB was formed again. This suggests that the stochastic region disappeared. However $m / n=2 / 1$ magnetic island might exist inside $\imath / 2 \pi=0.5$ rational surface because the locally flat $T_{e}$ profile was observed there. While, by cntr. ECCD, $\imath / 2 \pi$ in the core region was reduced and separated from 0.5 and the flattening of the $T_{e}$ profile did not occur there. Moreover the locally flat $T_{e}$ profile was not observed inside the $l / 2 \pi=0.5$ rational surface. The global stochasitization in the core region and generation of the magnetic island might able to be avoided by cntr. ECCD. The phase delay of the heat pulse is large inside the $r / 2 \pi=0.5$ rational surface. On the contrary, in the cases of co ECCD and additional ECRH, the phase delay is little inside the $l / 2 \pi=0.5$ rational surface. In the case of cntr. ECCD, compared to the case of co ECCD, less power is required to form and sustain the e-ITB and the region of the improvement confinement is wider.

\section{Summary}

Increase of the stored energy was observed in over-dense plasmas during $1 \mathrm{MW}$ EC wave injection. Profiles of $\Delta T_{e}$, and $\Delta\left(d T_{e} / d t\right)$ suggest the power absorption in the core region. Since the plasma cutoff is located near the plasma edge, the possible mechanism of heating is ECRH by EBW excited via the $\mathrm{O}-\mathrm{X}-\mathrm{B}$ mode conversion process. For precise analysis with ray-tracing to determine the power absorption region and heating efficiency, it is necessary to give the electron density profile in the peripheral region precisely because the plasma cutoff and the UHR layer is located very close to the plasma edge.

It was derived that the local modification of the rotational transform by ECCD affects the formation and sustainment of the e-ITB. If the width of the magnetic island can be changed by regulation of ECCD, it is expected that the influence of the magnetic island on the formation and sustainment of the e-ITB can be analyzed precisely and systematically in the same magnetic field configuration.

\section{Acknowledgements}

The authors appreciate the technical staff of ECH and LHD for their great efforts. This work was mainly performed under the budget code NIFS11ULRR701, 801 and partly supported by a grant for scientific research from the Ministry of Education, Science and Culture of Japan (C 23561006).

\section{References}

1. H. Igami, S. Kubo, H.P. Laqua, K. Nagasaki et. al. Rev. Sci. Instrum., 77, 10E931 (2006)

2. N. Ohyabu, T. Morisaki, S. Masuzaki, R. Sakamoto et. al. Phys. Rev. Lett. 97, 055002 (2006)

3. H. Igami, Y. Yoshimura, S. Kubo, T. Shimozuma et. al. Nucl. Fusion 49, 115005 (2009)

4. Y. Yoshimura et. al. Plasma Science ant Technology (to be published)s

5. H. Igami, S. Kubo, T. Shimozuma, Y. Yoshimura, H. Takahashi et. al, Plasma and Fusion Research (to be published)

6. J. Preinhaelter and V. Kopecky J. Plasma Phys. 10, 1 (1973)

7. E. Mjølhus J. Plasma Phys. 31 7(1984)

8. H. Igami, R. Ikeda, H. Takahashi, et. al. Plasma Science ant Technology 11, 430 (2009)

9. T. Shimozuma, S. Kubo, H. Idei, S. Inagaki, N. Tamura et al. Nucl. Fusion 45, 1396 (2005)

10. H. Takahashi, T. Shimozuma, S. Kubo et al. Proc. of 23rd IAEA FEC Conf., EXC/P8-15 (2010)

11. K. Ida et. al. Rev. Sci. Instrum. 76, 053505 (2005).

12. T. Ido et. al., Fusion Sci. Technol. 58436 (2010)

13. K. Ida et. al. Phys. Rev. Lett. 100045003 (2008) 